\title{
Osteitis Fibrosa Cystica of Tibia as Initial Manifestation of Primary Hyperparathyroidism
}

Shushan Shweta Jayker, Chippagi Jyothi Prakash, Manjunatha Yedatore Arkeswara, Mohammed Naushad Ansari, Shingati Muhammed Hashim, Parthasarathi Achappa

Department of Pathology, Rajarajeswari Medical College and Hospital, Kambipura, Mysore road, Bangalore-560074, Karnataka, India.

Corresponding Author:

Dr. Shushan Shweta Jayker

Email: shwetajayker@gmail.com

This is an Open Access article distributed under the terms of the Creative Commons Attribution License (creativecommons.org/ licenses/by/3.0)

Published

\section{July 22, 2016}

November 29, 2016

January 5, 2017

\begin{abstract}
Background: Primary hyperparathyroidism is disease of excessive secretion of parathyroid hormone. Osteitis fibrosa cystica or brown tumor, complication of primary hyperthyroidism is characterized by fibrotic cystic bony changes. Skeletal manifestations in the form of brown tumors are rare occuring in less than $2 \%$ of patients suffering from any form of hyperparathyroidism. Case Report: We report a 40-year-old woman with lytic lesions in tibia, diagnosed as osteitis fibrosa cystica secondary to parathyroid adenoma. Conclusion: Patients with primary hyperparathyroidism are usually asymptomatic and clinical presentation of the lesion in tibia is rarely a first sign of the disease.
\end{abstract}

Keywords: Neoplasms, Osteitis Fibrosa Cystica, Parathyroid Hormone, Primary Hyperparathyroidism, Tibia.

\section{Introduction}

Osteitis fibrosa cystica is a skeletal disease related to long standing end-stage hyperparathyroidism and is sometimes referred to as "brown tumor" because of its reddish appearance. However, it is not a true neoplasm but rather a reactive osteolytic lesion of bone and may mimic other diseases such as giant cell tumor, multiple bony metastasis or multiple myeloma [1]. Primary hyperparathyroidism is defined as hypercalcemia from the overproduction of parathyroid hormone (PTH) by one or more hyperfunctioning parathyroid glands [2]. It can be caused by a solitary adenoma in $80 \%$ of patients, parathyroid hyperplasia in $15 \%$, multiple adenoma in $5 \%$ and parathyroid carcinoma in less than $5 \%$ of patients [1]. Here we report a patient with primary hyperparathyroidism caused by parathyroid adenoma with osteitis fibrosa cystica of tibia as an unusual first manifestation of the disease.

\section{Case Report}

A 40 year old female patient presented to the orthopaedic out-patient department complaining of pain and swelling in the middle part of right leg since last three months. Plain X-ray revealed a well-defined lytic lesion with multiple thin internal septations in the cortex of mid shaft of right tibia [Fig.1]. Possibility of osteoblastoma, aneurysmal bone cyst and non-ossifying fibroma were suggested. MRI showed an expansile multiloculated lytic lesion arising eccentrically from the cortex of antero-medial aspect of right tibia, possibly adamantinoma [Fig.2,3]. Fine needle aspiration cytology of the lesion showed features of giant cell lesion and further investigations with excision biopsy were suggested [Fig.4]. The biochemical investigations showed elevated serum calcium levels of $13.3 \mathrm{mg} / \mathrm{dL}$ (normal 8.5-10.5 $\mathrm{mg} / \mathrm{dL}$ ), serum phosphorus of $1.6 \mathrm{mg} / \mathrm{dL}$ (normal 
$2.7-4.5 \mathrm{mg} / \mathrm{dL})$, serum alkaline phosphatase of 622 $\mathrm{IU} / \mathrm{L}$ (normal 64-306 IU/L) and serum parathyroid hormone of $703.20 \mathrm{pg} / \mathrm{mL}$ (normal upto 11.5 $\mathrm{pg} / \mathrm{mL}$ ). Excision biopsy of the lesion showed diffuse proliferation of osteoclast-type giant cells, increased blood vessels and areas of haemorrhage [Fig.5]. Ultrasound scan of neck showed a welldefined hypoechoic lesion at inferior pole of right lobe of thyroid with increased vascularity. A possibility of right inferior parathyroid adenoma was suggested. MRI scan of neck confirmed the same findings with additional findings of multiple lytic lesions in the right clavicle, left scapula and right mandible. The Tc $99 \mathrm{~m}$ sestamibi scan showed increased uptake in right inferior parathyroid site [Fig.6].

Based on the above findings the diagnosis of primary hyperparathyroidism with osteitis fibrosa cystica due to parathyroid adenoma was considered. Right inferior parathyroidectomy was done and the histopathological examination confirmed the lesion as parathyroid adenoma [Fig.7]. Two weeks postoperatively, the serum levels of calcium and parathyroid hormone declined to their normal limits.

\section{Discussion}

Osteitisfibrosa cystica also known as osteitis fibrosa, osteodystrophia fibrosa, Von Recklinghausen's disease of bone, is a skeletal disorder caused by prolonged exposure of bone to elevated parathyroid hormone levels in primary hyperparathyroidism [3]. Osteitis fibrosa cystica was first reported by von Recklinghausen in 1891; it presents as the end stage findings of hyperparathyroidism. However recently, with the technical development of imaging and laboratory screening methods, hypercalcemia due to primary or secondary hyperparathyroidism can often be detected early; as a result the frequency of osteitis fibrosa cystica has declined [1]. Lytic lesions caused by hyperparathyroidism are called brown tumors. The term "Brown tumor" is a misnomer because it is not a true neoplasm, but

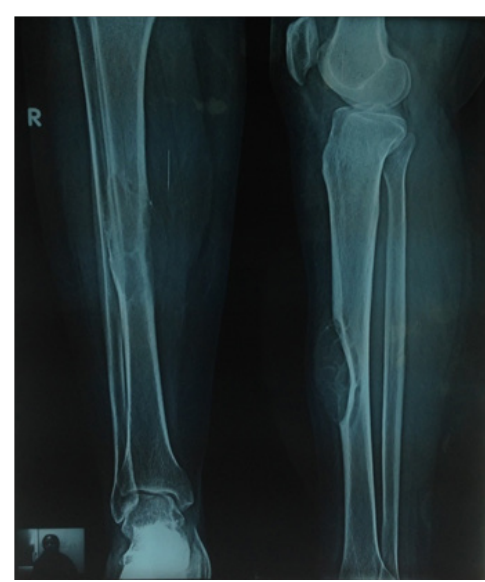

Fig.1: Well defined expansile lytic lesion involving the anterior cortex of mid shaft of tibia.
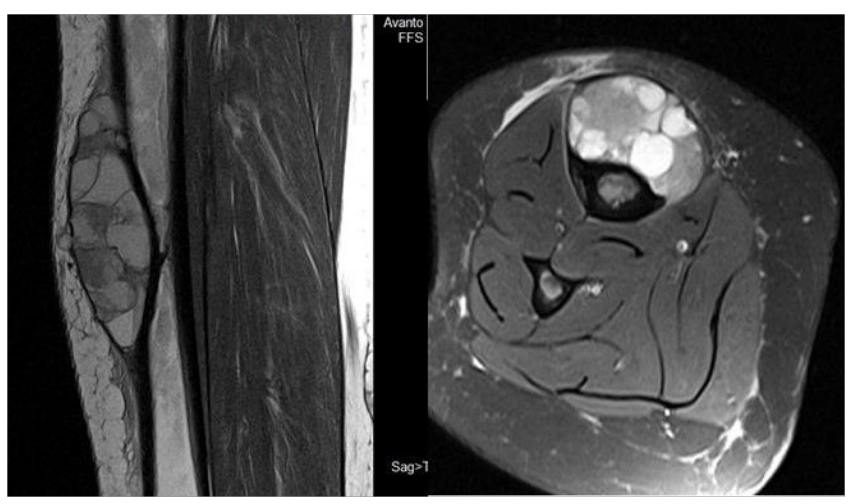

Fig.2: T2 sagittal and T2 FS axial MRI showing multiseptated heterogenous signal intensity lesion arising from cortex of tibia.

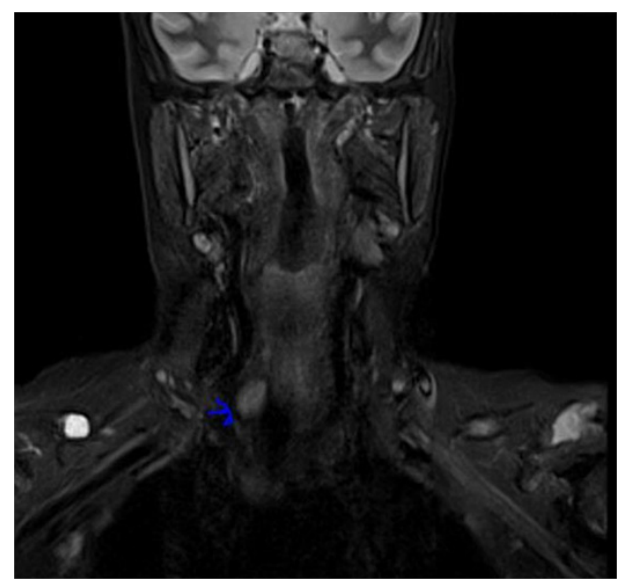

Fig.3: T2FS coronal MRI of neck showing hyper-intense parathyroid adenoma at the lower pole right thyroid gland. 


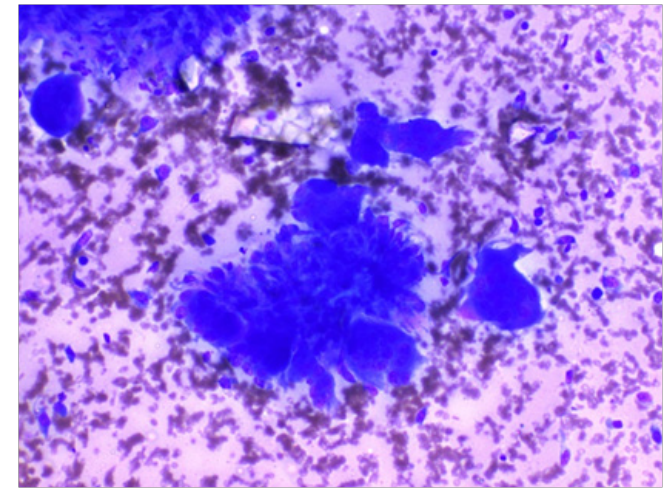

Fig.4: FNA smear showing many multinucleated giant cells admixed with stromal cells in a background of haemorrhage. (Giemsa, 20X).

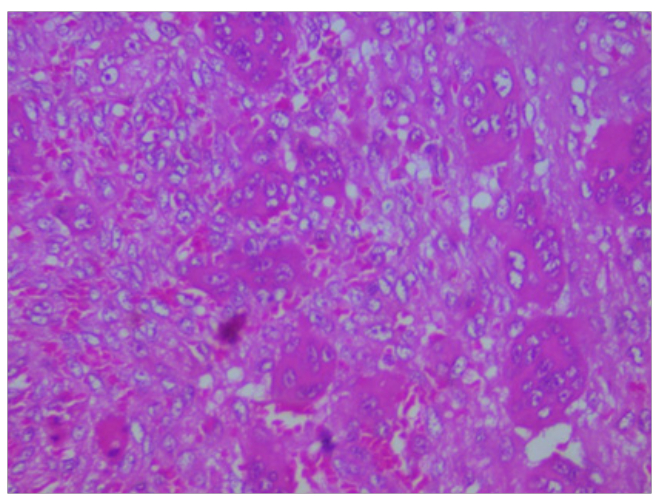

Fig.5: Section shows diffuse proliferation of osteoclasttype giant cells admixed with spindle cell proliferation and haemorrhage (H\&E, 20X).

benign hypervascular focal lesions in bones caused by increased osteoclastic activity and fibroblastic proliferation [4]. They are seen commonly in facial bones, mandible, sternum, pelvis, ribs, femur and rarely in vertebrae [3].

Primary hyperparathyroidism is characterized by the hyperfunctioning of parathyroid glands. The female:male ratio is $5: 2$. About $85 \%$ of cases are caused by a sporadic PTH secreting solitary adenoma of parathyroid chief cells. Multiglandular parathyroid hyperplasia occurs in $1-15 \%$ of patients with primary hyperparathyroidism. Parathyroid carcinoma is rare and occurs in less than $1 \%$ of cases. Although this condition can occur at any

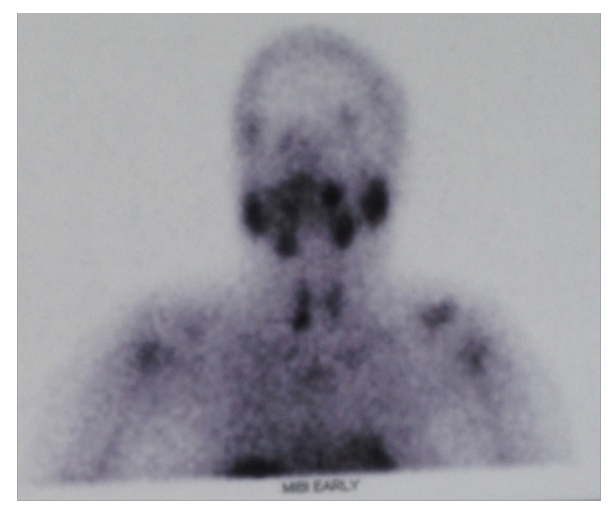

Fig.6: MIBI image showing focal increased radiotracer uptake in relation to lower pole of right lobe of thyroid.

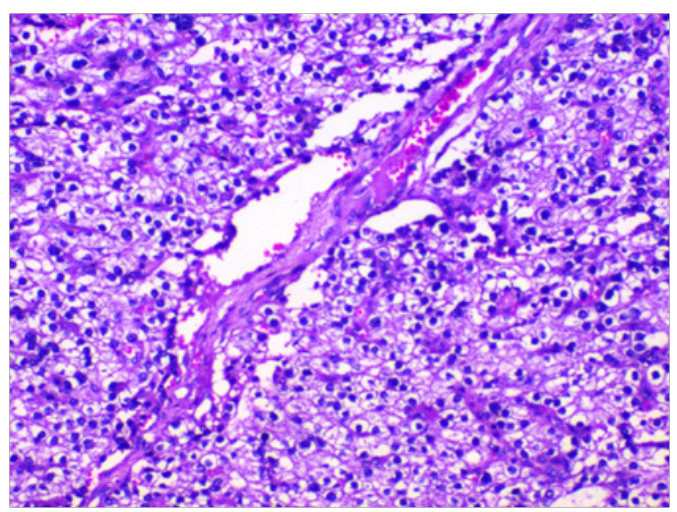

Fig.7: Section shows diffuse sheets of round to polygonal cells having clear cytoplasm and uniform nuclei (H\&E, 10X).

age, it commonly affects people over the age of 50 years and postmenopausal women $[5,6]$. Excessive secretion of PTH causes bone resorption accompanied by fibrovascular marrow replacement and increased osteoblastic activity; the imbalance of osteoclastic and osteoblastic activity manifest as a slow enlarging painful bony mass, osteitis fibrosa cystica, which can lead to pathological fractures. Fine needle aspiration biopsy (FNAB) of the masslike lesion, imaging studies, high serum calcium and PTH leves are useful for diagnosis. Treatment of osteitis fibrosa cystica caused by primary hyperparathyroidism is surgical removal of the parathyroid lesions. 


\section{Conclusion}

Osteitis fibrosa cystica has become a rare disease and lytic lesion of bone showing giant cells in histopathology should raise the suspicion of hyperparathyroidism. A high index of suspicion will lead to early diagnosis.

Contributors: All authors have contributed to patient management and manuscript writing.

Funding: None; Competing interests: None stated.

\section{References}

1. Lee HJ, Chung MS, Kim SH. Osteitis Fibrosa Cystica Mistaken for Malignant Disease. Clinical and Experimental Otorhinolaryngology. 2013;6(2):110-113.

2. Alhusban M, Baqain HZ. Mandibular brown tumor as the first manifestation of primary hyperparathyroidism: A case report. The Saudi Dental Journal. 2011;23:107109.

3. Abeysekera WYM, Kumara PMC, Amarasinghe AAWK, Fernando DMH, Perera ABS. Recurrent pathological fractures in osteitis fibrosa cystica: a case of undetected primary hyperparathyroidism due to parathyroid adenoma. The Sri Lanka Journal of Surgery. 2013;31(1):26-29.

4. Ullah E, Ahmad M, Ali SA, Redhu N. Primary hyperparathyroidism having multiple Brown tumors mimicking malignancy. Indian $\mathrm{J}$ Endocr Metab. 2012;16:1040-1042.

5. Pallan S, Rahman OM, Khan AA. Diagnosis and management of primary hyperparathyroidism. BMJ. 2012;344:e1013.

6. MacKenzie-Feder J, Sirrs S, Anderson D, Sharif J, Khan A. Primary Hyperparathyroidism: An Overview. Int J Endocrinol. 2011;2011:251410. 